\title{
Existence of Multiple Positive Solutions for Third-Order Three-Point Boundary Value Problem
}

\author{
Qiufeng Chen1, Jianli Li² \\ ${ }^{1}$ Key Laboratory of High Performance Computing and Stochastic Information Processing (HPCSIP), Changsha, China \\ ${ }^{2}$ School of Mathematics and Statistics, Hunan Normal University, Changsha, China \\ Email: 995460263@qq.com
}

How to cite this paper: Chen, Q.F. and $\mathrm{Li}$, J.L. (2019) Existence of Multiple Positive Solutions for Third-Order Three-Point Boundary Value Problem. Journal of Applied Mathematics and Physics, 7, 1463-1472. https://doi.org/10.4236/jamp.2019.77098

Received: May 27, 2019

Accepted: July 15, 2019

Published: July 18, 2019

Copyright () 2019 by author(s) and Scientific Research Publishing Inc. This work is licensed under the Creative Commons Attribution International License (CC BY 4.0).

http://creativecommons.org/licenses/by/4.0/

\begin{abstract}
In this paper, we study the existence of positive solutions for a class of third-order three-point boundary value problem. By employing the fixed point theorem on cone, some new criteria to ensure the three-point boundary value problem has at least three positive solutions are obtained. An example illustrating our main result is given. Moreover, some previous results will be improved significantly in our paper.
\end{abstract}

\section{Keywords}

Third-Order Three-Point Boundary Value Problem, Fixed Point Theorem, Three Positive Solutions

\section{Introduction}

As we all know, the earliest boundary value problem studied is Dirichlet problem. We need to find the solution of Laplace equation. Boundary value problems are most common in physics, such as wave equation. With the development of boundary value problems, many scholars began to pay attention to the study of higher-order boundary value problems. The third-order three-point problems have a wide range of applications in the fields of mathematics and physics [1] [2] [3] [4] [5]. Many works on the third-order boundary value problems have been established. In [6] [7] [8] [9] [10], the authors have studied the third-order three-point boundary value problem and proved that the model has at least one positive solution. Recently, there have been many papers dealing with the positive solutions of boundary value problems for nonlinear differential equations with various boundary conditions. For example, Anderson [11] obtained some 
existence results for positive solutions for the following system:

$$
\left\{\begin{array}{l}
x^{\prime \prime \prime}(t)=f(x(t)), 0<t<1 \\
x(0)=x^{\prime}\left(t_{2}\right)=x^{\prime \prime}(1)=0
\end{array}\right.
$$

where $f: R \rightarrow R$ is continuous, $f$ is nonnegative for $x \geq 0$ and $\frac{1}{2} \leq t_{2}<1$.

Moreover, Yao [12] considered the following system:

$$
\left\{\begin{array}{l}
u^{\prime \prime \prime}(t)+\lambda f(t, u(t))=0,0<t<1, \\
u(0)=u^{\prime}(0)=u(1)=0,
\end{array}\right.
$$

where $\lambda>0, f(t, u)$ is a Caratheodory function. The author proved that (1.2) has at least one positive solution by Krasnoselskii fixed point theorems.

With the development of third-order boundary value problems, Guo et al. [2] considered the existence of a positive solution to the third-order three-point boundary value problem as follows

$$
\left\{\begin{array}{l}
u^{\prime \prime \prime}(t)+a(t) f(u(t))=0, t \in(0,1) \\
u(0)=u^{\prime}(0)=0 . u^{\prime}(1)=\alpha u^{\prime}(\eta)
\end{array}\right.
$$

where $f:[0,+\infty) \rightarrow[0,+\infty)$ is continuous; $a:[0,1] \rightarrow[0,+\infty)$ is continuous and not identically zero on $\left[\frac{\eta}{\alpha}, \eta\right], 0<\eta<1$ and $1<\alpha<\frac{1}{\eta}$. By using the Guo-Krasnoselskii fixed point theorem, they proved that the system (1.3) has at least one positive solution.

To our best knowledge, few papers can be found in the literature for three positive solutions of third-order three-point boundary value problems. Motivated greatly by the above-mentioned excellent works, in this paper, we will consider the following model

$$
\left\{\begin{array}{l}
x^{\prime \prime \prime}(t)+h(t) f\left(t, x(t), x^{\prime}(t), x^{\prime \prime}(t)\right)=0,0<t<1, \\
x(0)=x^{\prime}(0)=0, x^{\prime}(1)=\xi x^{\prime}(\eta)
\end{array}\right.
$$

where $E=\left\{x \in C^{3}([0,1], R): x(0)=x^{\prime}(0)=0, x^{\prime}(1)=\xi x^{\prime}(\eta)\right\} \quad, \quad 0<\eta<1$, $1<\xi<\frac{1}{\eta}, f:[0,1] \times[0,+\infty) \times R \times R \rightarrow[0,+\infty)$ is a continuous function; $h:[0,1] \rightarrow[0,+\infty)$ is continuous and not identically zero on $\left[\frac{\eta}{\alpha}, \eta\right]$.

Obviously, this model is new because the nonlinear $f$ depends not only on the unknown function but also the derivative of unknown function. In particular, the system (1.2) is special case of system (1.4). By the properties of the Green's function, existence results of at least three positive solution for the third-order three-point boundary value problem are established by a new method which is different from the method in [13]. The paper is organized as follows. In Section 2, we present some notation and lemmas. In Section 3, we give the main results. In Section 4, an example is given to illustrate the main results of this 
paper.

\section{Preliminaries}

Definition 2.1. Let $E$ be a real Banach space. $K \subset E$ is a nonempty closed convex set. If it satisfies the following two conditions:

1) $x \in K, \lambda>0$ implies $\lambda x \in K$;

2) $x \in K,-x \in P$ implies $x=0$.

Then, $K$ is called a cone of $E$.

Definition 2.2. Suppose $K$ is a cone. The map $\alpha: K \rightarrow[0,+\infty)$ is continuous and satisfies the following inequality

$$
\alpha(t x+(1-t) y) \leq t \alpha(x)+(1-t) \alpha(y) \text { for any } x, y \in K \text { and } 0 \leq t \leq 1 .
$$

Then the map $\alpha$ is a nonnegative continuous convex function on $K$.

Suppose $K$ is a cone. The map $\varphi: K \rightarrow[0,+\infty)$ is continuous and satisfies the following inequality

$$
\varphi(t x+(1-t) y) \geq t \varphi(x)+(1-t) \varphi(y) \text { for any } x, y \in K \text { and } 0 \leq t \leq 1 .
$$

Then the map $\varphi$ is a nonnegative continuous concave function on $K$.

Lemma 2.1 [2] Assume $\xi \eta \neq 1$, then the system

$$
\left\{\begin{array}{l}
x^{\prime \prime \prime}(t)+y(t)=0,0<t<1, \\
x(0)=x^{\prime}(0)=0, x^{\prime}(1)=\xi x^{\prime}(\eta),
\end{array}\right.
$$

has a unique solution $x(t)=\int_{0}^{1} G(t, s) y(s) \mathrm{d} s$ for $y \in C[0,1]$,

where

$$
G(t, s)=\frac{1}{2(1-\xi \eta)}\left\{\begin{array}{l}
\left(2 t s-s^{2}\right)(1-\xi \eta)+t^{2} s(\xi-1), s \leq \min \{\eta, t\}, \\
t^{2}(1-\xi \eta)+t^{2} s(\xi-1), t \leq s \leq \eta, \\
\left(2 t s-s^{2}\right)(1-\xi \eta)+t^{2}(\xi \eta-s), \eta \leq s \leq t, \\
t^{2}(1-s), \max \{\eta, t\} \leq s .
\end{array}\right.
$$

If we denote $g(s)=\frac{1+\xi}{1-\xi \eta} s(1-s), s \in[0,1]$, then we have the following lemma.

Lemma 2.2 [2] Let $0<\eta<1,1<\xi<\frac{1}{\eta}$, then

1) $0 \leq G(t, s) \leq g(s)$, for any $(t, s) \in[0,1] \times[0,1]$;

2i) $G(t, s) \geq \beta g(s)$, for any $(t, s) \in\left[\frac{\eta}{\xi}, \eta\right] \times[0,1]$,

where $0<\beta=\frac{\eta^{2}}{2 \xi^{2}(1+\xi)} \min \{\xi-1,1\}<1$.

For positive real numbers $a, b, c, d$, we define the following convex sets:

$$
\begin{aligned}
& P(\gamma, d)=\{x \in K \mid \gamma(x)<d\}, \\
& P(\gamma, \varphi, b, d)=\{x \in K \mid b \leq \varphi(x), \gamma(x) \leq d\},
\end{aligned}
$$




$$
\begin{gathered}
P(\gamma, \alpha, \varphi, b, c, d)=\{x \in K \mid b \leq \varphi(x), \alpha(x) \leq c, \gamma(x) \leq d\}, \\
R(\gamma, \psi, a, d)=\{x \in K \mid a \leq \psi(x), \gamma(x) \leq d\} .
\end{gathered}
$$

Lemma 2.3 [14] (Arzela-Ascoli theorem) Let $\mu \subset C[0,1]$ be a operator, then $\mu$ is sequentially compact in $C[0,1]$ if and only if $\mu$ is uniformly bounded and equicontinuous.

Lemma 2.4 [5] (Krasnoselskii fixed point theorem) Let $E$ be a real Banach space. $K \subset E$ is a cone. Suppose $\gamma, \alpha$ are nonnegative continuous convex functions on $K . \varphi$ is a nonnegative continuous concave function on $K . \psi$ is a nonnegative continuous function on $K$, which satisfied $\psi(\lambda x) \leq \lambda \psi(x), \lambda \in[0,1]$ and for positive numbers of $q, d$, we have

$$
\varphi(x) \leq \psi(x),\|x\| \leq q \gamma(x), \forall x \in \overline{P(\gamma, d)} .
$$

Let $T: \overline{P(\gamma, d)} \rightarrow \overline{P(\gamma, d)}$ be a completely continuous operator. There exist positive numbers of $a, b, c$ and $a<b$ satisfing the following conditions:

1) $\{x \in P(\gamma, \alpha, \varphi, b, c, d) \mid \varphi(x)>b\} \neq \varnothing$, and $\alpha(T x)>b$, for all $x \in P(\gamma, \alpha, \varphi, b, c, d)$;

2) $\varphi(T x)>b$, for $x \in P(\gamma, \varphi, b, d)$, and $\alpha(T x)>c$;

3) $0 \notin R(\gamma, \psi, a, d)$, and $\psi(T x)<a$, for $x \in R(\gamma, \psi, a, d), \psi(x)=a$;

then $T$ has at least three fixed points $x_{1}, x_{2}, x_{3} \in \overline{P(\gamma, d)}$ such that

$$
\begin{gathered}
\gamma\left(x_{i}\right) \leq d, i=1,2,3 ; \quad b<\varphi\left(x_{1}\right) ; \\
a<\psi\left(x_{2}\right) \text {, as } \varphi\left(x_{2}\right)<b ; \psi\left(x_{3}\right)<a .
\end{gathered}
$$

\section{The Existence of Three Positive Solutions}

We define the norm

$$
\|x\|=\max \left\{\max _{t \in[0,1]}|x(t)|, \max _{t \in[0,1]}\left|x^{\prime}(t)\right|, \max _{t \in[0,1]}\left|x^{\prime \prime}(t)\right|\right\} .
$$

Define the cone by

$$
K=\left\{x \in E \mid x(t) \geq 0, t \in[0,1], \min _{t \in\left[\frac{\eta}{\xi}, \eta\right]} x(t) \geq \beta \max _{t \in[0,1]} x(t)\right\} .
$$

Suppose

$$
\gamma(x)=\max _{t \in[0,1]}\left|x^{\prime \prime}(t)\right|, \psi(x)=\alpha(x)=\max _{t \in[0,1]}|x(t)|, \varphi(x)=\min _{t \in\left[\frac{\eta}{\xi}, \eta\right]}|x(t)| .
$$

Lemma 3.1. Let $T: K \rightarrow E$ be the operator defined by

$$
T x(t)=\int_{0}^{1} G(t, s) h(s) f\left(s, x(s), x^{\prime}(s), x^{\prime \prime}(s)\right) \mathrm{d} s, x \in K .
$$

Then $T: K \rightarrow K$ is completely continuous.

Proof From the fact that $f$ is nonnegative continuous function and Lemma 2.2, we know that $T x(t) \geq 0, t \in[0,1]$. Let $x \in K$, from Lemma 2.2, we have

$$
\begin{aligned}
& \int_{0}^{1} G(t, s) h(s) f\left(s, x(s), x^{\prime}(s), x^{\prime \prime}(s)\right) \mathrm{d} s \\
& \leq \int_{0}^{1} g(s) h(s) f\left(s, x(s), x^{\prime}(s), x^{\prime \prime}(s)\right) \mathrm{d} s,
\end{aligned}
$$


so

$$
\begin{aligned}
\max _{t \in[0,1]}|T x(t)| & =\max _{t \in[0,1]}\left|\int_{0}^{1} G(t, s) h(s) f\left(s, x(s), x^{\prime}(s), x^{\prime \prime}(s)\right) \mathrm{d} s\right| \\
& \leq \int_{0}^{1} g(s) h(s) f\left(s, x(s), x^{\prime}(s), x^{\prime \prime}(s)\right) \mathrm{d} s .
\end{aligned}
$$

and

$$
\begin{aligned}
\min _{t \in\left[\frac{\eta}{\xi}, \eta\right]} T x(t) & =\min _{t \in\left[\frac{\eta}{\xi}, \eta\right]} \int_{0}^{1} G(t, s) h(s) f\left(s, x(s), x^{\prime}(s), x^{\prime \prime}(s)\right) \mathrm{d} s \\
& \geq \beta \int_{0}^{1} g(s) h(s) f\left(s, x(s), x^{\prime}(s), x^{\prime \prime}(s)\right) \mathrm{d} s \\
& \geq \beta \max _{t \in[0,1]}(T x)(t) .
\end{aligned}
$$

thus $T: K \rightarrow K$. According to the Arzela-Ascoli theorem, we prove that $T$ is a completely continuous operator.

For convenience, we note that

$$
\begin{aligned}
& A=\max \left\{\int_{0}^{1} h(s)\left|\frac{\partial^{2} G(t, s)}{\partial t^{2}}\right|_{t=0} \mathrm{~d} s, \int_{0}^{1} h(s)\left|\frac{\partial^{2} G(t, s)}{\partial t^{2}}\right|_{t=1} \mathrm{~d} s\right\}, \\
& B=\min \left\{\int_{\frac{\eta}{\xi}}^{\eta} G\left(\frac{\eta}{\xi}, s\right) h(s) \mathrm{d} s, \int_{\frac{\eta}{\xi}}^{\eta} G(\eta, s) h(s) \mathrm{d} s\right\}, \\
& C=\int_{0}^{1} g(s) h(s) \mathrm{d} s .
\end{aligned}
$$

Theorem 3.1. Suppose there exist $0<a<b<\frac{b}{\beta} \leq d$ such that

$\left(\mathrm{H}_{1}\right) \quad f(t, u, v, w) \leq \frac{d}{A},(t, u, v, w) \in[0,1] \times[0, d] \times[-d, d] \times[-d, d]$,

$\left(\mathrm{H}_{2}\right) \quad f(t, u, v, w)>\frac{b}{B},(t, u, v, w) \in\left[\frac{\eta}{\xi}, \eta\right] \times\left[b, \frac{b}{\beta}\right] \times[-d, d] \times[-d, d]$,

$\left(\mathrm{H}_{3}\right) \quad f(t, u, v, w)<\frac{a}{C},(t, u, v, w) \in[0,1] \times[0, a] \times[-d, d] \times[-d, d]$,

then the system (1.4) has at least three positive points $x_{1}, x_{2}$ and $x_{3}$ satisfying

$$
\begin{gathered}
\max _{t \in[0,1]}\left|x_{i}^{\prime \prime}(t)\right| \leq d, i=1,2,3 ; \\
\max _{t \in[0,1]}\left|x_{i}^{\prime}(t)\right| \leq d, i=1,2,3 ; \\
b<\min _{t \in\left[\frac{\eta}{\xi}, \eta\right]}\left|x_{1}(t)\right| ; \max _{t \in[0,1]}\left|x_{3}(t)\right|<a ; \\
a<\max _{t \in[0,1]}\left|x_{2}(t)\right| \leq \frac{b}{\beta}, \text { for } \min _{t \in\left[\frac{\eta}{\xi}, \eta\right]}\left|x_{2}(t)\right|<b .
\end{gathered}
$$

Proof For $x \in K$, we have

$$
x(t)=x(0)+\int_{0}^{t} x^{\prime}(s) \mathrm{d} s \leq t \max _{t \in[0,1]}\left|x^{\prime}(t)\right| \leq \max _{t \in[0,1]}\left|x^{\prime}(t)\right|
$$

so

$$
\max _{t \in[0,1]}|x(t)| \leq \max _{t \in[0,1]}\left|x^{\prime}(t)\right|
$$


Since

$$
x^{\prime}(t)=x^{\prime}(0)+\int_{0}^{t} x^{\prime \prime}(s) \mathrm{d} s
$$

which also implies that

$$
\left|x^{\prime}(t)\right| \leq \max _{t \in[0,1]}\left|x^{\prime \prime}(t)\right|
$$

therefore,

$$
\|x(t)\| \leq \gamma(x), x \in \overline{P(\gamma, d)}, \quad \beta \alpha(x) \leq \varphi(x) \leq \alpha(x)=\psi(x) .
$$

So we show that (1.7) of the Lemma 2.4 holds.

If $x \in \overline{P(\gamma, d)}$, we have $\gamma(x)=\max _{t \in[0,1]}\left|x^{\prime \prime}(t)\right| \leq d$.

And $T^{\prime \prime \prime} x(t)=-h(t) f\left(t, x(t), x^{\prime}(t), x^{\prime \prime}(t)\right) \leq 0$ for any $t \in[0,1]$. From assumption $\left(\mathrm{H}_{1}\right)$, we have $f\left(t, x(t), x^{\prime}(t), x^{\prime \prime}(t)\right) \leq \frac{d}{A}$, therefore,

$$
\begin{aligned}
\gamma(T x(t)) & =\max _{t \in[0,1]}\left|T^{\prime \prime} x(t)\right| \\
& =\max \left\{\left|T^{\prime \prime} x(1)\right|,\left|T^{\prime \prime} x(0)\right|\right\} \\
& \leq \max \left\{\int_{0}^{1}\left|\frac{\partial^{2} G(t, s)}{\partial t^{2}}\right|_{t=0} h(s) f\left(s, x(s), x^{\prime}(s), x^{\prime \prime}(s)\right) \mathrm{d} s,\right. \\
& \left.\int_{0}^{1}\left|\frac{\partial^{2} G(t, s)}{\partial t^{2}}\right|_{t=1} h(s) f\left(s, x(s), x^{\prime}(s), x^{\prime \prime}(s)\right) \mathrm{d} s\right\} \\
& \leq \frac{d}{A} A=d,
\end{aligned}
$$

hence

$$
T: \overline{P(\gamma, d)} \rightarrow \overline{P(\gamma, d)}
$$

Let $x(t)=\frac{b}{\beta}, t \in[0,1]$, it is easy to prove $x(t)=\frac{b}{\beta} \in P\left(\gamma, \alpha, \varphi, b, \frac{b}{\beta}, d\right)$, $\varphi(x)=\frac{b}{\beta}>b$, hence

$$
\left\{x \in P\left(\gamma, \alpha, \varphi, b, \frac{b}{\beta}, d\right) \mid \varphi(x)>b\right\} \neq \varnothing \text {. }
$$

If $x \in P\left(\gamma, \alpha, \varphi, b, \frac{b}{\beta}, d\right)$, then

$$
b \leq x(t) \leq \frac{b}{\beta},\left|x^{\prime}(t)\right| \leq d,\left|x^{\prime \prime}(t)\right| \leq d, t \in\left[\frac{\eta}{\xi}, \eta\right] .
$$

From assumption $\left(\mathrm{H}_{2}\right)$, we have $f\left(t, x(t), x^{\prime}(t), x^{\prime \prime}(t)\right)>\frac{b}{B}, t \in\left[\frac{\eta}{\xi}, \eta\right]$.

It can be divided into two situations: 
(i) $\varphi(T x)=T x\left(\frac{\eta}{\xi}\right)$

$$
\begin{aligned}
\varphi(T x) & =T x\left(\frac{\eta}{\xi}\right) \\
& =\int_{0}^{1} G\left(\frac{\eta}{\xi}, s\right) h(s) f\left(s, x(s), x^{\prime}(s), x^{\prime \prime}(s)\right) \mathrm{d} s \\
& >\frac{b}{B} \int_{\frac{\eta}{\xi}}^{\eta} G\left(\frac{\eta}{\xi}, s\right) h(s) \mathrm{d} s \geq b,
\end{aligned}
$$

(ii) $\varphi(T x)=T x(\eta)$,

$$
\begin{aligned}
\varphi(T x) & =T x(\eta) \\
& =\int_{0}^{1} G(\eta, s) h(s) f\left(s, x(s), x^{\prime}(s), x^{\prime \prime}(s)\right) \mathrm{d} s \\
& >\frac{b}{B} \int_{\frac{\eta}{\xi}}^{\eta} G(\eta, s) h(s) \mathrm{d} s \geq b,
\end{aligned}
$$

Therefore, we have $\varphi(T x)>b$ for $x \in P\left(\gamma, \alpha, \varphi, b, \frac{b}{\beta}, d\right)$, that is to say, condition (i) of Lemma 2.4 is satisfied.

Since $x \in P(\gamma, \varphi, b, d), \alpha(T x)>\frac{b}{\beta}$, we have

$$
\varphi(T x) \geq \beta \alpha(T x)>\beta \frac{b}{\beta}=b .
$$

Thus condition (ii) of Lemma 2.4 is satisfied.

Obviously, $\psi(0)=0<a$, so $0 \notin R(\gamma, \psi, a, d)$. We assume $x \in R(\gamma, \psi, a, d)$ and $\psi(x)=a$ hold.

From assumption $\left(\mathrm{H}_{3}\right)$. we have

$$
\begin{aligned}
\psi(T x) & =\max _{t \in[0,1]}|T x(t)| \\
& =\max _{t \in[0,1]} \int_{0}^{1} G(t, s) h(s) f\left(s, x(s), x^{\prime}(s), x^{\prime \prime}(s)\right) \mathrm{d} s \\
& \leq \int_{0}^{1} g(s) h(s) f\left(s, x(s), x^{\prime}(s), x^{\prime \prime}(s)\right) \mathrm{d} s \\
& <\frac{a}{C} C=a .
\end{aligned}
$$

Thus condition (iii) of Lemma 2.4 is also satisfied. From the above facts, the proof of Theorem 3.1 is completed.

\section{Example}

Example 4.1 Consider the following boundary value problem

$$
\left\{\begin{array}{l}
x^{\prime \prime \prime}(t)+4 t f\left(t, x(t), x^{\prime}(t), x^{\prime \prime}(t)\right)=0,0<t<1, \\
x(0)=x^{\prime}(0)=0, x^{\prime}(1)=\frac{6}{5} x^{\prime}\left(\frac{1}{4}\right),
\end{array}\right.
$$

where, 


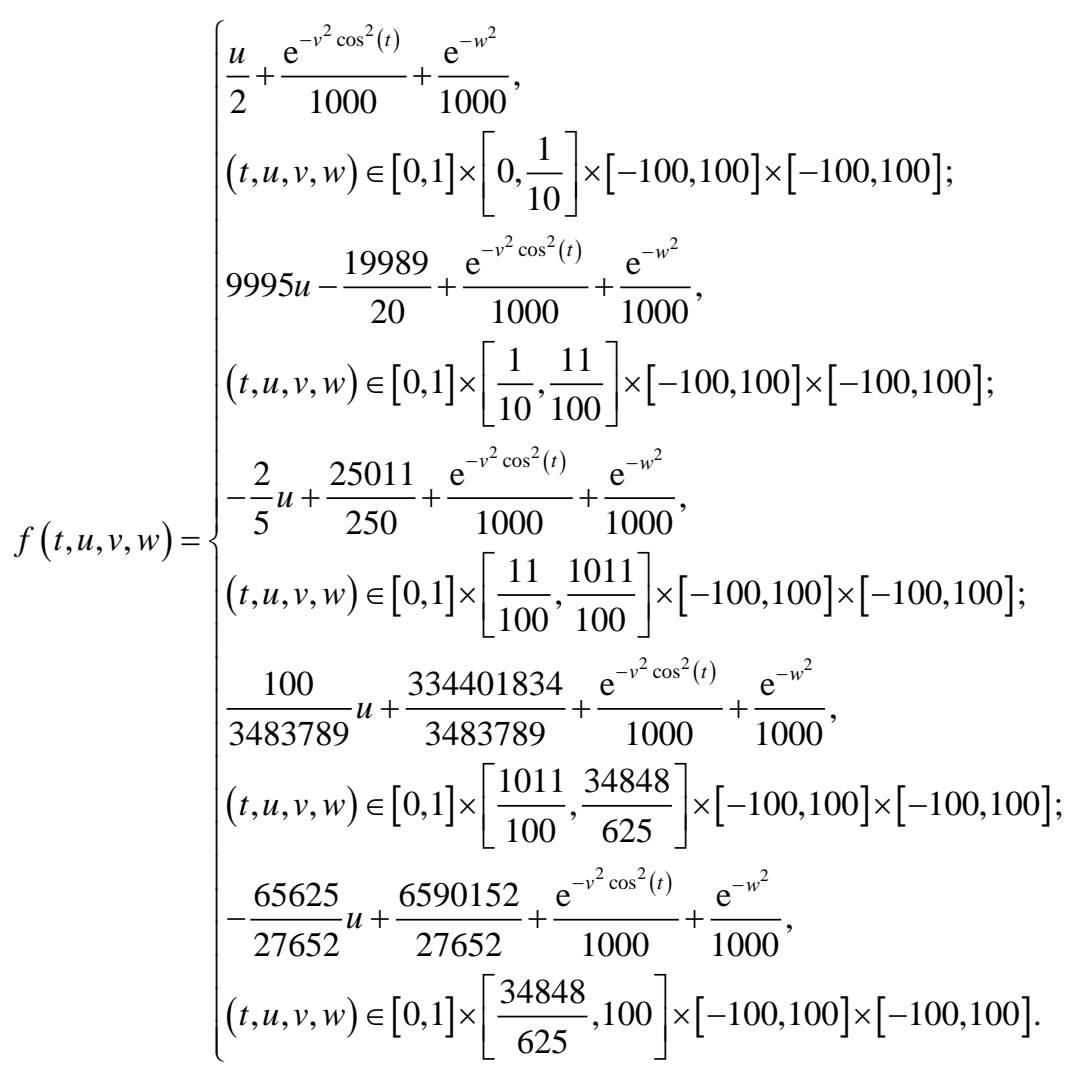

where $\xi=\frac{6}{5}, \eta=\frac{1}{4}, a=\frac{1}{10}, b=\frac{11}{100}, d=100$.

By the precise calculation, we have

$$
\begin{aligned}
& A=\frac{157}{168}, B=\frac{1375}{995328}, C=\frac{22}{21}, \frac{b}{\beta}=\frac{34848}{625}, \\
& f(t, u, v, w) \leq 106<\frac{d}{A} \approx 107, \\
& (t, u, v, w) \in[0,1] \times[0,100] \times[-100,100] \times[-100,100], \\
& f(t, u, v, w)>96>\frac{b}{B} \approx 79.63, \\
& (t, u, v, w) \in\left[\frac{5}{24}, \frac{1}{4}\right] \times\left[\frac{11}{100}, \frac{34848}{625}\right] \times[-100,100] \times[-100,100], \\
& f(t, u, v, w)<0.052<\frac{a}{C} \approx 0.09, \\
& (t, u, v, w) \in[0,1] \times\left[0, \frac{1}{10}\right] \times[-100,100] \times[-100,100] .
\end{aligned}
$$

All the conditions of theorem 3.1 are satisfied, so there are at least three positive solutions for the system.

\section{Conclusion}

In this paper, applying the fixed point theorem on the cone, we investigate the existence of positive solutions for a class of third-order three-point boundary 
value problem, which is a more general system. We obtain that the boundary value problem has at least three positive solutions.

\section{Conflicts of Interest}

The authors declare no conflicts of interest regarding the publication of this paper.

\section{References}

[1] Yao, Q. (2003) The Existence and Multiplicity of Positive Solutions for a Third-Order Three-Point Boundary Value Problem. Acta Mathematicae Applicatae Sinica, 19, 117-122. https://doi.org/10.1007/s10255-003-0087-1

[2] Boucherif, A. and Al-Malki, N. (2007) Nonlinear Three-Point Third Order Boundary Value Problems. Applied Mathematics and Computation, 190, 1168-1177. https://doi.org/10.1016/j.amc.2007.02.039

[3] Li, S. (2006) Positive Solutions of Nonlinear Singular Third-Order Two-Point Boundary Value Problem. Journal of Mathematical Analysis and Applications, 323, 413-425. https://doi.org/10.1016/j.jmaa.2005.10.037

[4] Yang, C., Liu, W., Shi, X. and Chen, H. (2009) Existence and Multiplicity of Monotone Positive Solutions for a Class of Third-Order Two-Point Boundary Value Problems. Mathematics in Practice and Theory, 39, 177-181.

[5] Sang, Y. and Wei, Z. (2011) Existence of Solutions to a Semipositone Third-Order Three-Point BVP on Time Scales. Acta Mathematica Scientia, 31, 455-465.

[6] Guendouz, C., Haddouchi, F. and Benacicha, S. (2018) Existence of Positive Solutions for a Nonlinear Third-Order Integral Boundary Value Problem. Annals of the Academy of Romanian Scientists. Series on Mathematics and Its Applications, 10, $1-11$.

[7] Guo, L., Sun, J. and Zhao, Y. (2008) Existence of Positive Solutions for Nonlinear Third-Order Three-Point Boundary Value Problems. Nonlinear Analysis, 68, 3151-3158. https://doi.org/10.1016/j.na.2007.03.008

[8] Guo, L. (2016) Existence of Two Positive Solutions for Third-Order Three-Point Boundary Value Problems of Nonlinear Differential Equations. Journal of Beihua University (Natural Science), 17, 566-571. https://doi.org/10.1186/s13661-016-0577-8

[9] Ping, J., Peng, J., Guo, L. and Zhao, H. (2009) Positive Solutions of a Class of Nonlinear Third-Order Three-Point Boundary Value Problems. Journal of Lanzhou University of Technology, 35, 139-142. https://doi.org/10.1155/2009/572512

[10] Sun, Y. (2008) Existence of Triple Positive Solutions for a Third-Order Three-Point Boundary Value Problem. Journal of Computational and Applied Mathematics, 211, 194-201. https://doi.org/10.1016/j.cam.2007.10.064

[11] Anderson, D.R. (1998) Multiple Positive Solutions for a Three-Point Boundary Value Problem. Mathematical and Computer Modelling, 27, 49-57. https://doi.org/10.1016/S0895-7177(98)00028-4

[12] Yao, Q. (2010) A Class of Third-Order Semipositone Boundary Value Problems with Caratheodory Nonlinear Term. Journal of Natural Science of Heilongjiang University, 27, 565-568.

[13] Wu, H. and Zhou, S. (2017) Existence of Positive Solutions to a Singular Third-Order 
Three-Point Boundary Value Problem. Annals of Applied Mathematics, 33, 186-193.

[14] Yosida, K. (1978) Functional Analysis. 4th Edition, Springer-Verlag, Berlin, Heidelberg, New York. 